\title{
Effect of nutrition on the body temperature and relative organ weights of broilers
}

\section{Efeito de diferentes planos nutricionais sobre a temperatura corporal e peso relativo de orgãos em frangos de corte}

\author{
Julyana Machado da Silva Martins ${ }^{1 *}$; Evandro de Abreu Fernandes ${ }^{2}$; \\ João Paulo Rodrigues Bueno ${ }^{3}$; Carolina Magalhães Caires Carvalho ${ }^{3}$; \\ Fernanda Heloisa Litz ${ }^{3}$; André Lucas Silva Masculi; Naiara Simarro Fagundes ${ }^{5}$; \\ Marina Cruvinel Assunção Silva ${ }^{6}$; Márcia Marques Silveira ${ }^{7}$; Cíntia Amaral Moraes ${ }^{6}$
}

\begin{abstract}
The objective of this study was to evaluate the effect of different nutritional plans on the body temperature and organ biometrics in male and female broilers, of two ages. Here, 1,700 birds were used (850 males and 850 females) in a completely randomized design composed of five treatments (- 3\%, $1.5 \%$, reference, $+1.5 \%$ and $+3 \%$ ), with 10 repetitions, totaling 50 experimental units; the reference treatment based on nutritional and energy levels indicated in previous studies was calculated from this. At 35 and $42 \mathrm{~d}$, the temperatures of the wing, head, shin, back, and cloaca in males and females were measured separately, and the average surface and body temperature were calculated. At $42 \mathrm{~d}$, relative weights of the gizzard, liver, heart, and small intestine were calculated. The temperatures of the wings, back, and cloaca, and consequently the average surface temperature and body temperatures, were not affected by nutritional plans. Effects of increasing the nutritional and energy levels were observed on liver weights, the gizzard, and the small intestine. We conclude that the nutritional plans did not affect body temperature. Males had higher body temperatures than females. Body temperature increased with increase in age, and the increase in the nutritional plans increased liver weight and reduced the gizzard weights.
\end{abstract}

Key words: Animal nutrition, body temperature, digestive system, poultry

\footnotetext{
${ }^{1}$ Discente do Curso de Doutorado em Zootecnia, Escola de Veterinária e Zootecnia, Universidade Federal de Goiás, UFG, Goiânia, GO, Brasil. E-mail: julyanamachado zoo@hotmail.com

${ }^{2}$ Prof. Dr., Faculdade de Medicina Veterinária, FAMEV, Universidade Federal de Uberlândia, UFU, Uberlândia, MG, Brasil. E-mail: evandrof@umuarama.ufu.br

${ }_{3}^{3}$ Discentes do Curso de Doutorado em Ciências Veterinárias, FAMEV, UFU, Uberlândia, MG, Brasil. E-mail: jprbueno@hotmail. com; carollcaires@yahoo.com.br; fernandalitz@veterinaria.med.br

${ }^{4}$ Discente do Curso de Graduação em Medicina Veterinária, FAMEV, UFU, Uberlândia, MG, Brasil. E-mail: andre.masculi@ hotmail.com

${ }^{5}$ Discente do Curso de Doutorado em Ciência Animal e Pastagens, Dept ${ }^{\circ}$ de Zootecnia, Escola Superior de Agricultura "Luiz de Queiroz", Universidade de São Paulo, ESALQ, Piracicaba, SP, Brasil. E-mail: nsfagundes@hotmail.com

${ }^{6}$ Mestres em Ciências Veterinárias, FAMEV, UFU, Uberlândia, MG, Brasil.E-mail: marinacruvinel@hotmail.com; moraescintia@, yahoo.com.br

7 Discente do Curso de Doutorado em Genética e Bioquímica, Instituto de Genética e Bioquímica, UFU, Uberlândia, MG, Brasil. E-mail: marciavet2003@yahoo.com.br

* Author for correspondence
} 


\title{
Resumo
}

\begin{abstract}
Objetivou-se avaliar a temperatura corporal e o peso relativo de órgãos em frangos de corte, machos e fêmeas, em duas idades, submetidos a diferentes planos nutricionais. Foram utilizadas 1700 aves (850 machos e 850 fêmeas) distribuídas em um delineamento inteiramente casualizado, composto de cinco tratamentos $(-3 \%,-1.5 \%$, referência, $+1.5 \%$ e $+3 \%)$ com 10 repetições, totalizando 50 unidades experimentais; sendo o tratamento referência baseado nos níveis nutricionais e energéticos recomendados por Rostagno et al. (2011) e os demais calculados a partir deste. Aos 35 e 42 dias de idade foram mensuradas as temperaturas da asa, cabeça, canela, dorso e da cloaca nos machos e fêmeas separadamente, e após calculou-se a temperatura superficial média e corporal. Aos 42 dias de idade também foram calculados os pesos relativos da moela, fígado, coração e intestino delgado. As temperaturas de asa, dorso e cloaca não foram afetadas pelos planos nutricionais, e consequentemente as temperaturas superficial média e corporal. Observou-se efeito do aumento dos níveis nutricionais e energéticos nos pesos do fígado, moela e do intestino delgado. Conclui-se que os planos nutricionais não influenciaram a temperatura corporal. Os machos apresentaram maior temperatura corporal que as fêmeas. A temperatura corporal se elevou conforme aumentou a idade. O aumento dos planos nutricionais aumentou o peso do fígado e reduziu o peso da moela.
\end{abstract}

Palavras-chave: Aves, ambiência animal, nutrição animal, sistema digestório

\section{Introduction}

The thermoneutral zone in birds depends on a number of intrinsic variables, including internal insulation and autonomic vasomotor mechanisms, and extrinsic variables, including room temperature and physical characteristics of facilities. Therefore, some handling alternatives for those variables exist, aimed at reducing the impact of stress on birds to improve thermal homeostasis (MACARI et al., 2004).

During feeding, nutrient metabolism causes increase in heat. The increment increases with the amount of food consumed and is inversely proportional to the energy content of the diet. Increased carbohydrate content provides greater heat increase increments, but in diets with oils substituted for carbohydrates, there is a lower heat increment. Proteins provide one of the highest heat increases, depending on the form that is provided by the feed ingredients (intact), due to a series of complex reactions required in their metabolism. Thus, the increment is reduced when synthetic amino acids are substituted for protein in the diet. Research has shown that environmental factors can effect metabolizable energy in broiler diets due to changes in food consumption (LONGO et al., 2006;
OLIVEIRA NETO et al., 2000), and subsequently protein and digestible amino acids (CHENG et al., 1997; OLIVEIRA et al., 2010, 2011; TAVERNARI et al., 2013), and the modification of these levels in the diet can also reduce the effects on body temperature.

Temperatures (surface and body temperature) in poultry serve as a physiological response to inadequate housing conditions (NASCIMENTO et al., 2011). Thus, it is important to determine the influence of different nutritional plans on body temperature variation, since the nutrient density of the diet has great influence on it. It is known that the body and surface/body temperatures of poultry also vary with sex (ALVES, 2012; AMARAL et al., 2011; MACARI et al., 2004), and age (CANGAR et al., 2008; MACARI; FURLAN, 2001; MARCHINI et al., 2007; SILVA et al., 2003).

It is possible that the nutritional and energy density of diets can influence digestive system development, and according to Lawrence and Fowler (2002) visceral tissues have a higher capacity for size reduction during malnutrition and, consequently, reduce their metabolic activities more effectively compared to carcass tissues. 
The objective of this study was to evaluate the effect of different nutritional plans on body temperature and relative weight of organs in male and female broiler chickens at two ages.

\section{Materials and Methods}

The experiment was conducted from MarchApril 2013, at the Broiler's Experimental Farm Fazenda Glória - Federal University of Uberlândia in Uberlândia, Minas Gerais, Brazil. All procedures in this study were performed as Registry Protocol CEUA/UFU 002/13 approved by the Ethics Committee on the use of animals, of the Federal University of Uberlândia.

A total of 1,700 broilers (850 males and 850 females) from a single age flock of the Hubbard Flex breed were raised from 1-42 d during the study.

They were housed in a brick shed with dimensions of $60 \times 10 \mathrm{~m}$, with a metal roof, tile asbestos cement, lined with plastic specific for poultry, a concrete floor, sides with short masonry walls and wire mesh screens of $4 \mathrm{~cm}^{2}$. Internally the shed was equipped with 80 pens of PVC pipes and galvanized wire mesh, each measuring $1.90 \times 1.50$ $\mathrm{m}$, fans on the both sides and water sprinklers on the ceiling to control the temperature, side curtains, and artificial lighting. Each pen was equipped with an automatic child drinker, a pendulum-shaped drinker, and a tubular feeder. Shavings were used as bedding material.

For temperature measurements, a completely randomized design with split plot in time $5 \times 2 \times$ 2 (five treatments, two sexes, and two ages) with 10 repetitions was used. Ages were evaluated at d 35 and 42, by analyzing two birds from each pen (one male and one female), totaling 20 animals per treatment.

To analyze the performance of the organs, the experiment was conducted in a completely randomized design in a factorial arrangement (2 $x$ 5), with two sexes and five treatments with five repetitions, with each bird being considered as one repetition, resulting in a total of 50 birds being used for analysis.

The treatments consisted of different nutritional plans, following the recommendation of Rostagno et al. (2011): 3 and 1.5\% below and 1.5 and $3 \%$ above the nutritional and energy levels of the reference plan, respectively (Tables 1, 2, 3 and 4). The reference plan was assumed as $0 \%$, where the other plans were raised or lowered in relation to this.

A feeding program based on four phases was used according to the age of the birds: the pre-starter phase (1-7 d), initial phase (8-21 d), fattening (22$35 \mathrm{~d})$, and slaughtering (36-42 d). The diets were formulated and prepared with sorghum, soybean meal, degummed soybean oil, dicalcium phosphate, limestone, salt, vitamin complex, minerals, and additives. As the density was increased, the amount of oil was also increased.

The management of the birds throughout the experiment followed the criteria of the Experimental Farm model to ensure proper ambience at every stage of life, clean and fresh water supply, and ad libitum feed. The birds were vaccinated against Marek's and Gumboro's disease in the hatchery and revaccinated against Gumboro's disease by the administration of the vaccine via drinking water at $12 \mathrm{~d}$ of age.

The lighting program inside the shed was divided into three phases, considering natural and artificial light, throughout the experiment. Phase 1 (1-7 d): $22 \mathrm{~h}$ of light, phase $2(8-21 \mathrm{~d}): 20 \mathrm{~h}$ of light, and phase 3 ( $22 \mathrm{~d}$ to flock withdrawal): $23 \mathrm{~h}$ of light.

To provide thermal comfort for the birds, the temperature was monitored daily every $3 \mathrm{~h}$ using three thermo-hygrometers (Data Logger Incoterm ${ }^{\circledR}$ ) installed at three different points of the shed $30 \mathrm{~cm}$ above the avian bed (Table 5). 
Table 1. Ingredients, composition percentage, and calculated feed values of the pre-starter diet (age of the birds, 1-7 days).

\begin{tabular}{lccccc}
\hline \multicolumn{1}{c}{ Ingredients } & \multicolumn{5}{c}{ Nutritional Plans } \\
\cline { 2 - 6 } & $3 \%$ below & $1.5 \%$ below & Reference & $1.5 \%$ above & $3 \%$ above \\
\hline Sorghum (\%) & 57.08 & 55.22 & 53.43 & 50.78 & 48.70 \\
Soybean meal (\%) & 35.58 & 36.38 & 37.18 & 38.77 & 39.77 \\
Soybean oil (\%) & 3.20 & 4.16 & 5.09 & 6.15 & 7.13 \\
Dicalcium phosphate (\%) & 1.80 & 1.80 & 1.85 & 1.90 & 1.90 \\
Limestone (\%) & 0.89 & 0.93 & 0.92 & 0.90 & 0.96 \\
Salt (\%) & 0.44 & 0.46 & 0.46 & 0.46 & 0.48 \\
Premix (\%) & 0.40 & 0.40 & 0.40 & 0.40 & 0.40 \\
L-Lysine (\%) & 0.31 & 0.32 & 0.33 & 0.30 & 0.30 \\
DL-Methionine (\%) & 0.19 & 0.21 & 0.22 & 0.22 & 0.23 \\
L-Threonine (\%) & 0.11 & 0.12 & 0.12 & 0.12 & 0.13 \\
Total (\%) & 100.00 & 100.00 & 100.00 & 100.00 & 100.00 \\
\hline Composition & & & & & 3.090 \\
Metabolizable Energy (kcal kg-1) & 2.910 & 2.955 & 3.000 & 3.045 & 23.19 \\
Crude Protein (\%) & 22.06 & 22.30 & 22.52 & 22.86 & 0.95 \\
Calcium (\%) & 0.89 & 0.91 & 0.92 & 0.93 & 0.48 \\
Available Phosphorus (\%) & 0.46 & 0.46 & 0.47 & 0.48 & 0.23 \\
Sodium (\%) & 0.21 & 0.22 & 0.22 & 0.22 & 1.36 \\
Digestible lysine (\%) & 1.28 & 1.30 & 1.32 & 1.34 & 0.69 \\
Digestible Methionine (\%) & 0.65 & 0,66 & 0.67 & 0.68 & 1.07 \\
Methionine + cystine (\%) & 0.92 & 1.02 & 1.04 & 1.06 & 0.88 \\
Digestible threonine (\%) & 0.83 & 0.85 & 0.86 & 0.87 & 0.27 \\
Digestible Tryptophan (\%) & 0.25 & 0.25 & 0.26 & 0.26 & 1.44 \\
Digestible Arginine (\%) & 1.36 & 1.38 & 1.40 & 1.42 & \\
\hline
\end{tabular}

* Initial Premix (composition per kg of product): Vitamin A 1,600,000.00 IU; vitamin B1 600.00 mg; vitamin B12 2,000.00 mcg; vitamin B2 $800.00 \mathrm{mg}$; vitamin B6 $400.00 \mathrm{mg}$; vitamin D3 400,000.00 IU; Vitamin E 3,000.00 mg; vitamin K $400.00 \mathrm{mg}$; zinc $12.60 \mathrm{~g}$; copper $1260.00 \mathrm{mg}$; selenium $80.00 \mathrm{mg}$; iron $10.50 \mathrm{~g}$; iodine $252.00 \mathrm{mg}$; manganese $12.60 \mathrm{~g}$; folic acid $140.00 \mathrm{mg}$; pantothenic acid $1600.00 \mathrm{mg}$; zinc bacitracin $11.00 \mathrm{~g}$; biotin $12.00 \mathrm{mg}$; choline $70.00 \mathrm{~g}$; methionine $336.60 \mathrm{~g}$; monensin sodium $22.00 \mathrm{~g}$; niacin $6000.00 \mathrm{mg}$.

For proper maintenance of temperature at all stages of rearing, equipment, including bell jars, fans, and sprinklers, beyond the curtain management, were used. The bells were used to heat the birds for the first $2 \mathrm{~d}$ at an average temperature of $32{ }^{\circ} \mathrm{C}$, which was reduced by $1{ }^{\circ} \mathrm{C}$ every $2 \mathrm{~d}$; between 7-8 $\mathrm{d}$, the bells were no longer used. In the following weeks, when temperatures above the ideal comfort zone for birds were observed, ventilation and sprinklers were used.

At 35 and $42 \mathrm{~d}$ old, a female and a male from each pen (repetition) were aleatory chosen to measure the temperatures of the wing (Twing), head (Thead), shin (Tshin), and back (Tback) using a digital infrared thermometer (Instrutemp DT8530 model), and cloaca using a mercury thermometer (model L185 Incoterm ${ }^{\circledR} / 06$ ). 
Table 2. Ingredients, composition percentage, and calculated feed values of the initial diet (age of the birds, 8-21 days).

\begin{tabular}{lccccc}
\hline \multirow{2}{*}{ Ingredients } & \multicolumn{5}{c}{ Nutritional plans } \\
\cline { 2 - 6 } & $3 \%$ below & $1.5 \%$ below & Reference & $1.5 \%$ above & $3 \%$ above \\
\hline Sorghum (\%) & 59.00 & 57.12 & 55.27 & 53.14 & 50.40 \\
Soybean meal (\%) & 32.95 & 33.75 & 34.57 & 35.66 & 37.27 \\
Soybean oil (\%) & 4.36 & 5.35 & 6.31 & 7.33 & 8.42 \\
Dicalcium phosphate (\%) & 1.44 & 1.44 & 1.50 & 1.55 & 1.55 \\
Limestone (\%) & 0.91 & 0.96 & 0.94 & 0.93 & 0.98 \\
Salt (\%) & 0.44 & 0.46 & 0.47 & 0.46 & 0.49 \\
Premix (\%)* & 0.40 & 0.40 & 0.40 & 0.40 & 0.40 \\
L-Lysine (\%) & 0.27 & 0.28 & 0.28 & 0.27 & 0.24 \\
DL-Methionine (\%) & 0.14 & 0.15 & 0.17 & 0.17 & 0.17 \\
L-Threonine (\%) & 0.09 & 0.09 & 0.09 & 0.09 & 0.08 \\
Total (\%) & 100.00 & 100.00 & 100.00 & 100.00 & 100.00 \\
\hline Composition & & & & & 3.147 \\
Metabolizable Energy (kcal kg ${ }^{-1}$ ) & 3.007 & 3.054 & 3.100 & 21.69 & 22.00 \\
Crude Protein (\%) & 20.91 & 21.13 & 21.37 & 0.85 & 0.86 \\
Calcium (\%) & 0.81 & 0.83 & 0.84 & 0.41 & 0.41 \\
Available Phosphorus (\%) & 0.39 & 0.39 & 0.40 & 0.22 & 0.23 \\
Sodium (\%) & 0.21 & 0.22 & 0.22 & 1.24 & 1.26 \\
Digestible lysine (\%) & 1.18 & 1.20 & 1.22 & 0.62 & 0.63 \\
Digestible Methionine (\%) & 0.59 & 0.60 & 0.61 & 0.89 & 0.91 \\
Methionine + cystine (\%) & 0.85 & 0.87 & 0.88 & 0.80 & 0.81 \\
Digestible threonine (\%) & 0.77 & 0.78 & 0.79 & 0.24 & 0.25 \\
Digestible Tryptophan (\%) & 0.23 & 0.24 & 0.24 & 1.34 & 1.36 \\
Digestible Arginine (\%) & 1.28 & 1.30 & 1.32 & & \\
\hline
\end{tabular}

* Initial Premix (composition per kg of product): vitamin A 1,600,000.00 IU; vitamin B1 600.00 mg; vitamin B12 2,000.00 mcg; vitamin B2 $800.00 \mathrm{mg}$; vitamin B6 $400.00 \mathrm{mg}$; vitamin D3 400,000.00 IU; vitamin E 3,000.00 mg; vitamin K $400.00 \mathrm{mg}$; zinc $12.60 \mathrm{~g}$; cooper $1260.00 \mathrm{mg}$; selenium $80.00 \mathrm{mg}$; iron $10.50 \mathrm{~g}$; iodine $252.00 \mathrm{mg}$; manganese $12.60 \mathrm{~g}$; folic acid $140.00 \mathrm{mg}$; pantothenic acid $1600.00 \mathrm{mg}$; zinc bacitracin $11.00 \mathrm{~g}$; biotin $12.00 \mathrm{mg}$; choline $70.00 \mathrm{~g}$; methionine $336.60 \mathrm{~g}$; monensin sodium $22.00 \mathrm{~g}$; niacin $6000.00 \mathrm{mg}$.

To obtain the average surface temperature (AST), the following formula was adopted, which was first described by Richards (1971) and adapted by Malheiros et al. (2000):

AST $=0,12$ Twing $+0,03$ Thead $+0,15$ Tshin + 0,70 Tback

The cloaca temperature (Tcloaca) and superficial AST were used to calculated the body temperature
(BT) as described by Richards (1971):

$$
\mathrm{BT}=0,3 \mathrm{AST}+0,7 \mathrm{Tcloaca}
$$

At $42 \mathrm{~d}$ old, 10 birds per treatment (five males and five females) were selected, representing the live weight equal to the average weight $( \pm 5 \%)$ of birds belonging to their respective treatment. Birds were identified by plastic numbered seals and then were fasted for $12 \mathrm{~h}$, receiving only water. 
Table 3. Ingredients, composition percentage and calculated feed values of the fattening diet (age of the birds, 22-35 days).

\begin{tabular}{lccccc}
\hline \multirow{2}{*}{ Ingredients } & \multicolumn{5}{c}{ Nutritional plans } \\
\cline { 2 - 5 } & $3 \%$ below & $1.5 \%$ below & Reference & $1.5 \%$ above & $3 \%$ Above \\
\hline Sorghum (\%) & 61.48 & 59.66 & 57.67 & 55.53 & 52.77 \\
Soybean meal (\%) & 29.64 & 30.44 & 31.38 & 32.45 & 34.10 \\
Soybean oil (\%) & 5.44 & 6.44 & 7.44 & 8.47 & 9.59 \\
Dicalcium phosphate (\%) & 1.20 & 1.26 & 1.28 & 1.31 & 1.30 \\
Limestone (\%) & 0.90 & 0.89 & 0.89 & 0.90 & 0.91 \\
Salt (\%) & 0.44 & 0.44 & 0.44 & 0.44 & 0.47 \\
Premix (\%) * & 0.40 & 0.40 & 0.40 & 0.40 & 0.40 \\
L-Lysine (\%) & 0.28 & 0.26 & 0.26 & 0.26 & 0.22 \\
DL-Methionine (\%) & 0.14 & 0.14 & 0.16 & 0.16 & 0.16 \\
L-Threonine (\%) & 0.08 & 0.07 & 0.08 & 0.08 & 0.08 \\
Total (\%) & 100.00 & 100.00 & 100.00 & 100.00 & 100.00 \\
\hline Composition & & & & & 3.296 \\
Metabolizable Energy (kcal kg ${ }^{-1}$ ) & 3.104 & 3.152 & 3.200 & 3.248 & 20.85 \\
Crude Protein (\%) & $1 ., 59$ & 19.77 & 20.07 & 20.37 & 0.78 \\
Calcium (\%) & 0.74 & 0.75 & 0.76 & 0.77 & 0.36 \\
Available Phosphorus (\%) & 0.34 & 0.34 & 0.35 & 0.36 & 0.22 \\
Sodium (\%) & 0.20 & 0.21 & 0.21 & 0.21 & 1.16 \\
Digestible lysine (\%) & 1.10 & 1.11 & 1.13 & 1.15 & 0.59 \\
Digestible Methionine (\%) & 0.55 & 0.56 & 0.57 & 0.58 & 0.84 \\
Methionine + cystine (\%) & 0.80 & 0.81 & 0.82 & 0.83 & 0.76 \\
Digestible threonine (\%) & 0.72 & 0.73 & 0.74 & 0.75 & 0.23 \\
Digestible Tryptophan (\%) & 0.21 & 0.22 & 0.22 & 0.22 & 1.24 \\
Digestible Arginine (\%) & 1.18 & 1.20 & 1.22 & & 1.26 \\
\hline
\end{tabular}

* Fattening Premix (composition per kg of product): vitamin A 1,280,000.00 IU; vitamin B1 400.00 mg; vitamin B12 1,600.00 mcg; vitamin B2 720.00 mg; vitamin B6 320.00 mg; vitamin D3 350,000.00 IU; vitamin E 2,400.00 mg; vitamin K 300.00 mg; copper $1200.00 \mathrm{mg}$; iron $10.00 \mathrm{~g}$; iodine $240.00 \mathrm{mg}$; manganese $12.00 \mathrm{~g}$; selenium $60.00 \mathrm{mg}$; zinc $12.00 \mathrm{~g}$; folic acid $100.00 \mathrm{mg}$; pantothenic acid1600.00 mg; biotin $6.00 \mathrm{mg}$; choline $50.00 \mathrm{~g}$; halquinol $6000.00 \mathrm{mg}$; methionine $267.30 \mathrm{~g}$; niacin $4800.00 \mathrm{mg}$; salinomicin $13.20 \mathrm{~g}$.

At the slaughterhouse, the birds were again weighed on a semi-analytical balance (Marte BL3200H) with an accuracy of $0.01 \mathrm{~g}$ and then slaughtered (stunning, bleeding, scalding, plucking, and gutting). Subsequently the gizzard, liver, heart, and small intestine were separated and weighed. The gizzard was previously opened by a longitudinal incision and all food contents removed. Thus, the relative weight was calculated based on the following formula:
Relative weight $(\%)=\frac{\text { Organ weight } \mathrm{x} 100}{\text { Body weight }}$

After the verification of normal data errors, the results were submitted to variance analysis with $5 \%$ significance and a regression was performed between nutritional plans, using the statistical program SAS 9.3 (SAS, 2011). 
Table 4. Ingredients, composition percentage and calculated feed values of the slaughtering diet (age of the birds, 36-42 days).

\begin{tabular}{lccccc}
\hline \multirow{2}{*}{ Ingredients } & \multicolumn{5}{c}{ Nutritional plans } \\
\cline { 2 - 5 } & $3 \%$ below & $1.5 \%$ below & Reference & $1.5 \%$ above & $3 \%$ above \\
\hline Sorghum (\%) & 64.08 & 62.24 & 60.55 & 57.82 & 55.69 \\
Soybean meal (\%) & 27.24 & 28.04 & 28.67 & 30.29 & 31.31 \\
Soybean oil (\%) & 5.70 & 6.69 & 7.67 & 8.79 & 9.85 \\
Dicalcium phosphate (\%) & 1.00 & 1.00 & 1.05 & 1.06 & 1.11 \\
Limestone (\%) & 0.77 & 0.79 & 0.79 & 0.80 & 0.79 \\
Salt (\%) & 0.42 & 0.45 & 0.45 & 0.45 & 0.47 \\
Premix (\%)* & 0.30 & 0.30 & 0.30 & 0.30 & 0.30 \\
L-Lysine (\%) & 0.26 & 0.25 & 0.26 & 0.23 & 0.22 \\
DL-Methionine (\%) & 0.17 & 0.18 & 0.19 & 0.20 & 0.20 \\
L-Threonine (\%) & 0.06 & 0.06 & 0.07 & 0.06 & 0.06 \\
Total (\%) & 100.00 & 100.00 & 100.00 & 100.00 & 100.00 \\
\hline Composition & & & & & 3.299 \\
Metabolizable Energy (kcal kg ${ }^{-1}$ ) & 3.153 & 3.201 & 3.250 & 19.55 & 19.83 \\
Crude Protein (\%) & 18.70 & 18.90 & 19.06 & 0.67 & 0.68 \\
Calcium (\%) & 0.64 & 0.65 & 0.66 & 0.31 & 0.32 \\
Available Phosphorus (\%) & 0.30 & 0.31 & 0.31 & 0.21 & 0.22 \\
Sodium (\%) & 0.20 & 0.21 & 0.21 & 1.08 & 1.09 \\
Digestible lysine (\%) & 1.03 & 1.04 & 1.06 & 0.54 & 0.55 \\
Digestible Methionine (\%) & 0.51 & 0.52 & 0.53 & 0.78 & 0.79 \\
Methionine + cystine (\%) & 0.75 & 0.76 & 0.77 & 0.70 & 0.71 \\
Digestible threonine (\%) & 0.67 & 0.68 & 0.69 & 0.21 & 0.22 \\
Digestible Tryptophan (\%) & 0.20 & 0.21 & 0.21 & 1.15 & 1.17 \\
Digestible Arginine (\%) & 1.11 & 1.13 & 1.14 & & \\
\hline
\end{tabular}

*Slaughtering Premix (composition per kg of product): vitamin A 1.300.260,00 IU; vitamin B1 166.00 mg; vitamin B12 1667.00 mcg; vitamin B2 666.80 mg; vitamin B6 200.00 mg; vitamin D3 400,000.00 IU; vitamin E 2167.10 mg; vitamin K 333.40 mg; copper $2000.00 \mathrm{mg}$; iron $16.60 \mathrm{~g}$; iodine $400.00 \mathrm{mg}$; manganese $20.00 \mathrm{~g}$; selenium $60.68 \mathrm{mg}$; zinc $20.00 \mathrm{~g}$; folic acid $100.00 \mathrm{mg}$; pantothenic acid $1333.00 \mathrm{mg}$; biotin $6.67 \mathrm{mg}$; choline $50.00 \mathrm{~g}$; methionine $230.00 \mathrm{~g}$; niacin $4000.00 \mathrm{mg}$; virginiamycin 3666.00 $\mathrm{mg}$.

Table 5. Minimum, average, and maximum temperature values (in degrees Celsius), recorded weekly recorded inside the shed, from 1-42 days, Uberlândia, Minas Gerais, in March and April of 2013.

\begin{tabular}{lccc}
\hline & Age & \multicolumn{3}{c}{ Temperature $\left({ }^{\circ} \mathrm{C}\right)$} \\
\cline { 2 - 4 } & Minimum & Average & Maximum \\
\hline $01-07 \mathrm{~d}$ & 27.1 & 31.2 & 33.8 \\
$08-14 \mathrm{~d}$ & 22.8 & 27.4 & 32.4 \\
$15-21 \mathrm{~d}$ & 21.8 & 26.4 & 30.8 \\
$22-28 \mathrm{~d}$ & 20.1 & 27.0 & 32.2 \\
$29-35 \mathrm{~d}$ & 20.0 & 26.6 & 30.8 \\
$36-42 \mathrm{~d}$ & 19.0 & 26.0 & 32.5 \\
\hline
\end{tabular}




\section{Results and Discussion}

The results obtained for temperatures of the shin, wing, head, and back are shown in Table 6. There was no interaction between nutrition plans, sex, and ages and no interaction between sexes and nutrition plans for any of the variables.

Table 6. Temperatures in degrees Celsius $\left({ }^{\circ} \mathrm{C}\right)$ of the shin (Tshin), wing (Twing), head (Thead) and back (Tback) of broilers, males and females, at 35 and 42 days of age, subjected to different nutritional plans.

\begin{tabular}{|c|c|c|c|c|c|}
\hline & & Tshin & Twing & Thead & Tback \\
\hline \multirow{5}{*}{ Plans } & $-3 \%$ & 37.49 & 32.47 & 34.77 & 32.09 \\
\hline & $-1.5 \%$ & 35.33 & 31.77 & 33.59 & 31.98 \\
\hline & 0 & 34.74 & 31.85 & 33.71 & 31.99 \\
\hline & $1.5 \%$ & 35.16 & 31.57 & 33.51 & 31.90 \\
\hline & $3 \%$ & 35.66 & 31.90 & 33.72 & 32.16 \\
\hline \multirow{2}{*}{ Gender } & Fêmeas & 34.67 & 32.03 & 33.71 & 31.64 \\
\hline & Machos & 36.38 & 31.80 & 34.01 & 32.41 \\
\hline \multirow{3}{*}{ Age } & 35 & 34.38 & 31.84 & 33.54 & 31.88 \\
\hline & 42 & 36.97 & 31.98 & 34.18 & 32.21 \\
\hline & $\mathrm{VC}(\%)$ & 9.09 & 5.27 & 5.27 & 5.09 \\
\hline \multirow{11}{*}{ Effect } & Plan & $<0.0001$ & 0.1778 & Quadratic & 0.9590 \\
\hline & Gender & 0.0001 & 0.3341 & 0.2235 & 0.0009 \\
\hline & Age & $<0.0001$ & 0.5541 & 0.0107 & 0.1983 \\
\hline & Plans x Gender & 0.4418 & 0.5980 & 0.8976 & 0.8393 \\
\hline & Females & $\mathrm{ns}$ & ns & ns & ns \\
\hline & Males & $\mathrm{ns}$ & ns & $\mathrm{ns}$ & ns \\
\hline & Plans x Age & $<0.0001$ & 0.7081 & 0.7155 & 0.2855 \\
\hline & 35 days old & Quadratic & ns & $\mathrm{ns}$ & ns \\
\hline & 42 days old & Cubic & ns & ns & ns \\
\hline & Gender x Age & 0.0753 & 0.8610 & 0.4529 & 0.0419 \\
\hline & Plans x Gender x Age & 0.0525 & 0.4306 & 0.8389 & 0.9012 \\
\hline
\end{tabular}

ns $=$ not significant $(\mathrm{P}>0.05)$

$\mathrm{VC}=$ Variation Coefficient.

There was a significant interaction between age and nutrition plans only for shin temperature, with a quadratic effect at $35 \mathrm{~d}$ and cubic effect at $42 \mathrm{~d}$, according to the equations $\mathrm{y}=0,3056 \times 2-0,617 \mathrm{x}+$ $33.002\left(R^{2}=0.9784\right)$ and $y=-0,1032 \times 3+0,1024 \times 2$ $+0,8972 \mathrm{x}+36.494\left(\mathrm{R}^{2}=0.971\right)$, respectively. As the plan to lower temperature at $35 \mathrm{~d}$ by $1.01 \%$. The greatest variation of shin temperature in relation to other surfaces was due to the lack of feathers in this region, making a change over a short time more likely (NÄÄS et al., 2010; NASCIMENTO et al.,
2011). According to Esmay (1969), in areas without feathers, a variation in the surface temperature at $20{ }^{\circ} \mathrm{C}$ occurs most due to vasomotor tone.

The effect of the nutritional plans for the temperatures of the shin and head, noting the quadratic effect on the temperature of the head, were determined using the equation $\mathrm{y}=0,0784 \times 2$ $0,146 x+33.508\left(R^{2}=0.8517\right)$, estimating a lower temperature for the plan of +0.931 . There was also a significant difference between the ages for Thead, which increased with increasing age. 
There were differences between males and females $(\mathrm{P}<0.05)$ for the temperatures of shin and back. The male temperatures were higher than the females. According to Silva et al. (2007), the difference of the back temperature can be explained by better feather coverage at the back of females compared to males. According to Amaral et al. (2011), males lose more heat to the surrounding environment, thereby increasing their metabolic heat production.

There was a significant interaction between sex and age in Tback (Table 7), as males at $35 \mathrm{~d}$ had a higher back temperature than at $42 \mathrm{~d}$. This was not the case with females, where the temperature did not differ between ages.

Table 7. Interaction between gender and age for back temperature of broilers, at 35 and 42 days of age, subjected to different nutritional plans.

\begin{tabular}{lccc}
\hline \multirow{2}{*}{ Gender } & \multicolumn{2}{c}{ Age } & \multirow{2}{*}{ P value } \\
\cline { 2 - 4 } & $35 \mathrm{~d}$ & $42 \mathrm{~d}$ & 0.5926 \\
\hline Females & 31.56 & 31.73 & 0.0192 \\
Males & 32.79 & 32.03 & \\
P value & 0.0002 & 0.3501 & \\
\hline
\end{tabular}

There was no interaction $(\mathrm{P}>0.05)$ between nutritional plans, sexes, and ages, or between sexes and plans for the surface, cloacal, and body temperatures (Table 8 ). Interaction between nutritional plans and ages for cloacal temperature, of a cubic effect to $42 \mathrm{~d}$ were observed, represented by the equation $\mathrm{y}=-0,0157 \mathrm{x} 3+0,0202 \mathrm{x} 2+0,0903 \mathrm{x}$ $+42.014\left(\mathrm{R}^{2}=0.965\right)$, which did not occur for surface and body temperatures.

Table 8. Average Surface Temperature (AST), cloacal temperature (Tcloaca) Average Body Temperature (ABT) in degrees Celsius $\left({ }^{\circ} \mathrm{C}\right)$ of males and females at 35 and 42 days of age, when subjected to different nutritional plans.

\begin{tabular}{lcccc}
\hline & & & & Continue $\ldots$ \\
\hline \multirow{4}{*}{ Plans } & $-3 \%$ & AST & Tcloaca & ABT \\
& $-1.5 \%$ & 33.02 & 42.00 & 39.31 \\
& 0 & 32.51 & 41.84 & 39.04 \\
\multirow{2}{*}{ Gender } & $1.5 \%$ & 32.44 & 41.93 & 39.09 \\
& $3 \%$ & 32.40 & 41.97 & 39.10 \\
\multirow{2}{*}{ Age } & Females & 32.70 & 41.88 & 39.12 \\
\hline \multirow{2}{*}{} & Males & 32.25 & 41.91 & 39.01 \\
& 35 & 32.98 & 41.94 & 39.25 \\
\hline & 42 & 32.50 & 41.75 & 38.97 \\
& $\mathrm{CV}(\%)$ & 32.52 & 42.10 & 39.29 \\
\hline
\end{tabular}




\begin{tabular}{ccccc}
\hline & & & ... Continuation \\
& Plans & 0.2071 & 0.3043 & 0.1864 \\
Gender & 0.0002 & 0.6424 & 0.0014 \\
Age & 0.2451 & $<0.0001$ & $<0.0001$ \\
Effect & Plans x Gender & 0.9636 & 0.8223 & 0.8877 \\
Females & $\mathrm{ns}$ & $\mathrm{ns}$ & $\mathrm{ns}$ \\
& Males & $\mathrm{ns}$ & $\mathrm{ns}$ & $\mathrm{ns}$ \\
& Plans x Age & 0.7135 & 0.0153 & 0.7731 \\
35 days old & $\mathrm{ns}$ & 0.4727 & $\mathrm{~ns}$ \\
42 days old & $\mathrm{ns}$ & Cúbico & $\mathrm{ns}$ \\
Gender x Age & 0.0265 & 0.9076 & 0.0715 \\
Plans x Gender x & 0.7739 & 0.9439 & 0.7596 \\
\hline
\end{tabular}

ns $=$ not significant $(\mathrm{P}>0.05)$

$\mathrm{VC}=$ Variation Coefficient.

Nutritional plans exerted no effect on the surface, cloacal, and body temperatures, probably because environmental temperature was constantly controlled to the thermal comfort range, and the birds had to make little effort to maintain thermal homeostasis. According to Furlan and Macari (2008), birds kept at thermoneutral temperature have a minimal metabolic rate and homeothermia is maintained with minimum energy expenditure.

Medeiros et al. (2005) reported that even if the temperature decreases to $20{ }^{\circ} \mathrm{C}$ or increases to $32{ }^{\circ} \mathrm{C}$, the variation in cloacal $\left(41,0-42,2{ }^{\circ} \mathrm{C}\right)$ and surface $\left(38,4-40.9^{\circ} \mathrm{C}\right)$ temperatures are still within ideal limits, which would not apply if the ambient temperature decreased to $16{ }^{\circ} \mathrm{C}$ or increased to 36 ${ }^{\circ} \mathrm{C}$, resulting in thermal stress.

Body temperature of birds increases when the ambient temperature rapidly reaches 30 ${ }^{\circ} \mathrm{C}$ (BOONE; HUGHES, 1971), but when the temperature gradually rises, the body temperature remains constant until the ambient temperature reaches $33{ }^{\circ} \mathrm{C}$ (WELKER et al., 2008).

Regarding the bird's gender, there was a significant effect $(\mathrm{P}<0.05)$ where males had higher AST and ABT temperatures than females. As the heat from metabolism produced by the animals varies according to gender, this result may be associated with intense metabolic activity, which is greater in male than female broilers throughout the growing period (ALVES, 2012; MACARI et al., 2004).

There was a higher cloacal and ABT $(\mathrm{P}<0.05)$ with age. This result was similar to several studies showing that cloacal and body temperature increases as birds become older (MACARI; FURLAN, 2001; SILVA et al., 2003; MARCHINI et al., 2007).

There was no significant interaction between gender and age, and average surface temperature (Table 9).

Table 9. Interaction between gender and age with average surface temperature of broiler chickens at 35 and 42 days of age, when subjected to different nutritional plans.

\begin{tabular}{lccc}
\hline \multirow{2}{*}{ Gender } & \multicolumn{2}{c}{ Age } & P value \\
\cline { 2 - 3 } & 35 days & 42 days & \\
\hline Females & 31.93 & 32.57 & 0.0171 \\
Males & 33.08 & 32.88 & 0.4488 \\
P value & $<0.0001$ & 0.2491 & \\
\hline
\end{tabular}


Males had a higher AST than females at $35 \mathrm{~d}$. The temperature of the males did not differ between ages. The interaction was due to lower AST in females at $35 \mathrm{~d}$ compared to females at $42 \mathrm{~d}$, and that was not noticed with males. This variable is very important since it is related to heat loss to the environment. According to Cangar et al. (2008), the variation in the surface temperature can be up to 8 ${ }^{\circ} \mathrm{C}$ between the first and last weeks. The increase in AST with increase in age was observed only for females, because close to the time of slaughter, they showed greater fat deposition than protein deposition (KESSLER et al., 2000), and fat acts as a cover that retains heat.
There was no significant interaction between gender and nutritional plans for the following variables: live weight, heart, liver, gizzard, and small intestine weights (Table 10). Gender only affected body weight and the gizzard and small intestine $(\mathrm{P}<0.05)$, with higher body weight seen for males and higher weight of the gizzard and small intestines seen for females. The nutritional plans did not affect liver and heart weights $(\mathrm{P}>$ 0.05); however, there was a significant effect on liver, gizzard, and small intestine weights. Ribeiro et al. (2001), Rocha et al. (2003), and Barbosa et al. (2008) found no differences in the heart weight of birds under different nutritional plans.

Table 10. Body weight and relative weights of the heart, liver, gizzard, and small intestine of male and female broilers, at 42 days of age, when subjected to different nutritional plans.

\begin{tabular}{ccccccc}
\hline & & Live weight $(\mathrm{kg})$ & Heart $(\%)$ & Liver $(\%)$ & Gizzard (\%) & Small Intestine (\%) \\
\hline \multirow{4}{*}{ Plans } & $-3 \%$ & 2.776 & 0.4689 & 1.7660 & 1.5148 & 2.5685 \\
& $-1.5 \%$ & 2.777 & 0.4937 & 1.8790 & 1.5052 & 2.9727 \\
& 0 & 2.788 & 0.4963 & 1.7244 & 1.4419 & 2.8276 \\
& $1.5 \%$ & 2.802 & 0.5028 & 1.9603 & 1.5113 & 3.1093 \\
\multirow{2}{*}{ Gender } & $3 \%$ & 2.800 & 0.4688 & 1.8261 & 1.3372 & 2.8568 \\
\hline \multirow{4}{*}{ Females } & 2.541 & 0.4803 & 1.8096 & 1.5020 & 2.9880 \\
& Males & 3.037 & 0.4919 & 1.8528 & 1.4221 & 2.7460 \\
\hline \multirow{4}{*}{ VC $(\%)$} & 9.52 & 13.65 & 8.47 & 9.32 & 12.12 \\
& Plans & 0.9523 & 0.7168 & Linear & Linear & Quadratic \\
& Gender & $<0.0001$ & 0.5576 & 0.2798 & 0.0259 & 0.0047 \\
& Interaction & 0.9622 & 0.3489 & 0.4483 & 0.5788 & 0.3210 \\
& Females & $\mathrm{ns}$ & $\mathrm{ns}$ & $\mathrm{ns}$ & $\mathrm{ns}$ & $\mathrm{ns}$ \\
& Males & $\mathrm{ns}$ & $\mathrm{ns}$ & $\mathrm{ns}$ & $\mathrm{ns}$ & $\mathrm{ns}$ \\
\hline
\end{tabular}

ns $=$ not significant $(\mathrm{P}>0.05)$

$\mathrm{VC}=$ Variation Coefficient.

The liver and gizzard have a lagged linear effect of the nutritional plans, represented by the equation $\mathrm{y}=0,0132 \mathrm{x}+1.8304\left(\mathrm{R}^{2}=0.8161\right)$ and $\mathrm{y}=-0,0233 \mathrm{x}$ $+1.4621\left(\mathrm{R}^{2}=0.5284\right)$, respectively, increased for the liver and decreased for the gizzard.

The increment of the nutritional levels may have increased liver metabolic function, and subsequently organ size. According to Xavier et al. (2008), the presence of fatty acids at high levels can stimulate an increase in the secretion of digestive enzymes, promoted by hypertrophy of the secretory cells, causing an increase in the liver. Aletor et al. (2003) suggest that, in high protein diets, the transport of metabolized lipids in the liver to other tissues may be increased by increased apolipoprotein synthesis, due to high availability of essential amino acids. 
According to Dozier et al. (2006), the dietary energy level controls the food intake of broilers, where higher available energy reduces food intake. Thus, this lower feed intake may have reduced the size of the gizzard, due to lower work done by that organ. According to Ribeiro et al. (2002), the mechanical work of the gizzard causes the greater development of the longitudinal smooth muscle of the organ, resulting in hypertrophy and increased muscle mass.

A similar result for gizzard weight was found by Rocha et al. (2003) with the Hubbard line, where the level of $3.000 \mathrm{kcal} \mathrm{kg}^{-1}$ of metabolizable energy showed a greater weight than the level of $2.850 \mathrm{kcal} \mathrm{kg}^{-1}$ for the treatments with $20 \%$ crude protein.

The weight of the small intestine was affected quadratically, and is shown by the equation $\mathrm{y}=$ $+-0,0281 \times 2+0,0475 x+2.9936\left(R^{2}=0.6662\right)$, estimating the higher weight to the plan $0.845 \%$. A similar result was found by Xavier et al. (2008) for broilers at $7 \mathrm{~d}$, when evaluating different energy levels in pre-starter feed.

The increase in energy density by adding more oil in the diets promotes greater retention time of the food in the digestive system because, according to Swenson and Reece (1996), dietary fat stimulates the release of cholecystokinin, which reduces the speed of emptying the digestive system that can stimulate an increase in its size.

\section{Conclusion}

The different nutritional plans did not affect body temperature of broilers. Males had higher temperatures than females, and body temperature increased with age.

The increase of both nutritional and energy levels in the plans resulted in a higher liver weight and a lower gizzard weight.

\section{References}

ALETOR, V. A.; EDER, K.; BECKER, K.; PAULICKS, B. R.; ROTH, F. X.; ROTH-MAIER, D. A. The effects of conjugated linoleic acids or an alpha-glucosidase inhibitor on tissue lipid concentrations and fatty acid composition of broiler chicks fed a low-protein diet. Poultry Science, Champaign, v. 82, n. 5, p. 796-804, 2003.

ALVES, F. M. S. Calor metabólico de frangos de corte e poedeiras alimentados com diferentes fontes lipídicas. 2012. Dissertação (Mestrado em Zootecnia) - Programa de Pós-Graduação em Zootecnia, Faculdade de Ciências Agrárias, Universidade Federal da Grande Dourados, Dourados.

AMARAL, A. G.; YANAGI JUNIOR, T.; LIMA, R. R.; TEIXEIRA, V. H.; SCHIASSI, L. Efeito do ambiente de produção sobre frangos de corte sexados criados em galpão comercial. Arquivo Brasileiro de Medicina Veterinária e Zootecnia, Belo Horizonte, v. 63, n. 3, p. 649-658, 2011.

BARBOSA, F. J. V.; LOPES, J. B.; FIGUEIRÊDO, A. V.; ABREU, M. L. T.; DOURADO, L. R. B.; FARIAS, L. A.; PIRES, J. E. P. Níveis de energia metabolizável em rações para frangos de corte mantidos em ambiente de alta temperatura. Revista Brasileira de Zootecnia, Viçosa, MG, v. 37, n. 5, p. 849-855, 2008.

BOONE, M. A.; HUGHES, B. L. Wind velocity as it affects body temperature, water consumption and feed consumption during heat stress of roosters. Poultry Science, Champaign, v. 50, n. 5, p.1535-1537, 1971.

CANGAR, Ö.; AERTS, J. M.; BUYSE, J.; BERCKMANS, D. Quantification of the spatial distribution of surface temperatures of broilers. Poultry Science, Champaign, v. 87, n. 12, p. 2493-2499, 2008.

CHENG, T. K.; HAMRE, M. L.; COON, C. N. Effect of environmental temperature, dietary protein, and energy levels on broiler performance. The Journal of Applied Poultry Research, Athens, v. 6, n. 1, p. 1-17, 1997.

DOZIER, W. A.; PRICE, C. J.; KIDD, M. T.; CORZO, A.; ANDERSON, J.; BRANTON, S. L. Growth performance, meat yield, and economic responses of broilers fed diets varying in metabolizable energy from thirty to fifty-nine days of age. The Journal of Applied Poultry Research, Athens, v. 15, n. 3, p. 367-382, 2006.

ESMAY, M. L. Principles of animal environmental. Westort Connecticut: AVI Publishing Company, 1969. $325 \mathrm{p}$. 
FURLAN, R. L.; MACARI, M. Termorregulação. In: MACARI, M.; FURLAN, R. L.; GONZALES, E. (Org.). Fisiologia aviária aplicada a frangos de corte. Jaboticabal: FUNEP, 2008. p. 209-230.

KESSLER, A. M.; SNIZEK JÚNIOR, P. N.; BRUGALLI, I. Manipulação da quantidade de gordura na carcaça de frangos. In: CONFERÊNCIA APINCO DE CIÊNCIA E TECNOLOGIA AVÍCOLA, 18., 2000, Campinas. Anais... Campinas: FACTA, 2000. p. 23-25.

LAWRENCE, T. L. J.; FOWLER, V. R. Growth of farm animals. Aberdeen: CAB International, 2002. 368 p.

LONGO, F. A.; SAKOMURA, N. K.; RABELLO, C. B. V.; FIGUEIREDO, A. N.; FERNANDES, J. B. K. Exigências energéticas para mantença e para o crescimento de frangos de corte. Revista Brasileira de Zootecnia, Viçosa, MG, v. 35, n. 1, p. 119-125, 2006.

MACARI, M.; FURLAN, R. L. Ambiência na produção de aves de corte. In: SILVA, I. J. O. (Org.). Ambiência na produção de aves em clima tropical. Piracicaba: FUNEP, 2001. p. 31-87.

MACARI, M.; FURLAN, R. L.; MAIORKA, A. Aspectos fisiológicos e de manejo para manutenção da homeostase térmica e controle de síndromes metabólicas. In: MENDES, A. A.; NÄÄS, I. A.; MACARI, M. (Org.). Produção de frangos de corte. Campinas: FACTA, 2004. p. 137-155.

MALHEIROS, R. D.; MORAES, V. M. B.; BRUNO, L. D. G.; MALHEIROS, E. B.; FURLAN, R. L.; MACARI, M. Environmental temperature and cloacal and surface temperatures of broiler chicks in first week post-hatch. The Journal of Applied Poultry Research, Athens, v. 9, n. 1, p. 111-117, 2000.

MARCHINI, C. F. P.; SILVA, P. L.; NASCIMENTO, M. R. B. M.; TAVARES, M. Frequência respiratória e temperatura cloacal em frangos de corte submetidos à temperatura ambiente cíclica elevada. Archives of Veterinary Science, Curitiba, v. 12, n. 1, p. 41-46, 2007.

MEDEIROS, C. M.; BAÊTA, F. C.; OLIVEIRA, R. F. M.; TINÔCO, I. F. F.; ALBINO, L. F. T.; CECON, P. R. Efeitos da temperatura, umidade relativa e velocidade do ar em frangos de corte. Engenharia na Agricultura, Viçosa, MG, v. 13, n. 4, p. 277-286, 2005.

NÄÄS, I. A.; ROMANINI, C. E. B.; NEVES, D. P.; NASCIMENTO, G. R.; VERCELLINO, R. A. Broiler surface temperature distribution of 42 day old chickens. Scientia Agrícola, Piracicaba, v. 67, n. 5, p. 497-502, 2010.

NASCIMENTO, G. R.; PEREIRA, D. F.; NÄÄS, I. A.; RODRIGUES, L. H. A. Índice Fuzzy de conforto térmico para frangos de corte. Engenharia Agrícola, Jaboticabal, v. 31, n. 2, p. 219-229, 2011.

OLIVEIRA NETO, A. R.; OLIVEIRA, R. F. M.; DONZELE, J. L.; ROSTAGNO, H. S.; FERREIRA, R. A.; MAXIMIANO, H. C.; GASPARINO, E. Efeito da temperatura ambiente sobre o desempenho e características de carcaça de frangos de corte alimentados com dieta controlada e dois níveis de energia metabolizável. Revista Brasileira de Zootecnia, Viçosa, MG, v. 29, n. 1, p. 183-190, 2000.

OLIVEIRA, W. P.; OLIVEIRA, R. F. M.; DONZELE, J. L.; ALBINO, L. F. T.; MARTINS, M. S.; MAIA, A. P. A. Redução do nível de proteína bruta em rações para frangos de corte em ambiente de termoneutralidade. Revista Brasileira de Zootecnia, Viçosa, MG, v. 40, n. 8, p. 1725-1731, 2011.

OLIVEIRA, W. P.; OLIVEIRA, R. F. M.; DONZELE, J. L.; GOMES, P. C.; MARTINS, M. S.; ASSIS, A. P. Redução do nível de proteína bruta em rações para frangos de corte em ambiente de estresse por calor. Revista Brasileira de Zootecnia, Viçosa, MG, v. 39, n. 5, p. 1092-1098, 2010.

RIBEIRO, A. M. L.; MAGRO, N.; PENZ JUNIOR, A. M. Granulometria do milho em rações de crescimento de frangos de corte e seu efeito no desempenho e metabolismo. Revista Brasileira de Ciência Avícola, Campinas, v. 4, n. 1, p. 1-7, 2002.

RIBEIRO, A. M. L.; PENZ JÚNIOR, A. M.; TEETER, R. G. Effects of 2-hydroxy-4-(methylthio) butanoic acid and DL-Methionine on broiler performance and compensatory growth after exposure to two different environmental temperatures. The Journal of Applied Poultry Research, Athens, v. 10, n. 4, p. 419-426, 2001.

RICHARDS, S. A. The significance of changes in the temperature of the skin and body core of the chicken in the regulation of heat loss. The Journal of Physiology, Cambridge, v. 216, n. 1, p. 1-10, 1971.

ROCHA, P. T.; STRINGHINI, J. H.; ANDRADE, M. A.; LEANDRO, N. S. M.; ANDRADE, M. L.; CAFÉ, M. B. Desempenho de frangos de corte alimentados com rações pré-iniciais contendo diferentes níveis de proteína bruta e energia metabolizável. Revista Brasileira de Zootecnia, Viçosa, MG, v. 32, n. 1, p. 162$170,2003$.

ROSTAGNO, H. S.; ALBINO, L. F. T.; DONZELE, J. L.; GOMES, P. C.; OLIVEIRA, R. F.; LOPES, D. C.; FERREIRA, A. S.; BARRETO, S. L. T.; EUCLIDES, R. F. Tabelas brasileiras para aves e suínos: composição de alimentos e exigências nutricionais. Viçosa MG: UFV, 2011. $252 \mathrm{p}$. 
STATISTICAL ANALYSIS SYSTEM - SAS. Statistical analysis system: user's guide: statistics. Version 9.3. Cary: SAS Institute Inc., 2011.

SILVA, M.A.N.;BARBOSAFILHO, J.A. D.; ROSÁRIO, M. F.; SILVA, C. J. M.; SILVA, I. J. O.; SAVINO, V. J. M.; COELHO, A. A. D. Fatores de estresse associados à criação de linhagens de avós de frangos de corte. Revista Brasileira de Zootecnia, Viçosa, MG, v. 36, n. 3, p. 652659, 2007.

SILVA, M. A. N.; HELLMEISTER FILHO, P.; ROSÁRIO, M. F.; COELHO, A. A. D.; SAVINO, V. J. M.; GARCIA, A. A. F.; SILVA, I. J. O.; MENTEN, J. F. M. Influência do sistema de criação sobre o desempenho, a condição fisiológica e o comportamento de linhagens de frangos para corte. Revista Brasileira de Zootecnia, Viçosa, MG, v. 32, n. 1, p. 208-213, 2003.
SWENSON, M. J.; REECE, W. O. Dukes: fisiologia dos animais domésticos. Rio de Janeiro: Guanabara, 1996. $856 \mathrm{p}$.

TAVERNARI, F. C.; LELIS, G. R.; VIEIRA, R. A.; ROSTAGNO, H. S.; ALBINO, L. F. T.; OLIVEIRA NETO, A. R. Valine needs in starting and growing Cobb (500) broilers. Poultry Science, Champaign, v. 92, n. 1, p. 151-157, 2013.

WELKER, J. S.; ROSA, A. P.; MOURA, D. J.; MACHADO, L. P.; CATELAN, F.; UTTPATEL, R. Temperatura corporal de frangos de corte em diferentes sistemas de climatização. Revista Brasileira de Zootecnia, Viçosa, MG, v. 37, n. 8, p. 1463-1467, 2008.

XAVIER, S. A. G.; STRINGHINI, J. H.; BRITO, A. B.; ANDRADE, M. A.; LEANDRO, N. S. M.; CAFÉ, M. B. Níveis de energia metabolizável em rações pré-iniciais para frangos de corte. Revista Brasileira de Zootecnia, Viçosa, MG, v. 37, n. 1, p. 109-115, 2008. 